MODELING, IDENTIFICATION AND CONTROL, 1993, vOL. 14, NO. 2, 59-72

doi:10.4173/mic.1993.21

\title{
Dynamics and control of unstable distillation columns $\dagger$
}

\author{
ELLING W. JACOBSEN $\$ \S$ and SIGURD SKOGESTAD $\ddagger$
}

Keywords: Distillation, multiple steady states, instability, limit cycles.

The fact that ideal two-product distillation columns may have multiple steady states and unstable operating points has only recently been recognized. This paper discusses the dynamics and control implications. It is shown that columns operating with reflux and boilup as independent variables become unstable if the internal flows are sufficiently large. An open-loop unstable operating point may be stabilized by use of one-point control, i.e., feedback control of a column composition or temperature. If the control is not sufficiently tight, the column may go into a stable limit cycle. Finally, it is shown that with distillate flow and boilup as independent variables the operating points may become unstable if the level control is not sufficiently tight. The column may also in this case go into a stable limit cycle.

\section{Introduction}

Distillation is undoubtedly the most studied unit operation in the process control literature. However, in all previous studies the column dynamics have been assumed to be asymptotically stable (with level and pressure loops closed). The main reason is that most authors have considered dynamic models with constant molar flows (neglected energy balance) and in addition assumed the inputs (e.g., reflux and boilup) to be given on a molar basis.

For the case of molar inputs there are several papers on uniqueness and asymptotic stability of the operating points in homogeneous distillation. Most papers treat the constant molar flow case (neglected energy balance), for example Lapidus and Amundson 1950, Acrivos and Amundson 1955, Rosenbrock 1960, 1962. Doherty and Perkins (1982) provide a review of results published in this area, and conclude that, for constant molar flows, multiplicity and instability is impossible for single-staged 'columns' and any multistage column separating a binary mixture. Sridhar and Lucia (1989) include the energy balance in the model and conclude under certain assumptions that also in this general case binary distillation columns will exhibit unique and stable solutions. However, they only study a limited set of specifications, namely $Q_{D} Q_{B}$ and $L B$.

However, in a recent paper Jacobsen and Skogestad (1991a) report two kinds of multiplicity which may occur in distillation.

(1) Jacobsen and Skogestad argue that only in rare cases do columns under operation have all the manipulated inputs on a molar basis. For instance, fixing the valve position will normally correspond closely to fixing the geometric average of mass and volumetric flow-rate. As they show, the transformation

Received 10 September 1992.

† This paper has previously been presented at DYCORD +92, Maryland, and is reprinted with permission from Pergamon Press.

‡ Chemical Engineering, University of Trondheim-NTH, N-7034 Trondheim, Norway. UK.

$\S$ Presently at Centre for Process Systems Engineering, Imperial College, London SW7 2BY, 
from mass- or volume flows to molar flows is nonlinear due to the composition dependence and may in some cases become singular, even for the binary case with constant molar flows. A singularity in the input transformation will imply that multiple solutions exist in terms of outputs (composition) for a given set of inputs (flows). One of the solutions will be unstable.

(2) In addition, Jacobsen and Skogestad show that when the energy balance is included in the model, even molar inputs may yield multiple solutions. Both types of multiplicity and instability may be experienced in industrial columns operated with inputs on a mass- or volume basis.

Jacobsen and Skogestad (1991a) treat the multiplicity from a steady-state point of view only. In this paper we study the dynamics and control of columns with multiple solutions. We limit ourselves mainly to discussing one control configuration (set of specifications), namely the case where mass reflux $L_{w}$ and molar boilup $V$ are used as independent variables. This is the most widespread configuration in industry, and is the configuration for which multiplicity and instability is most likely to occur (Jacobsen and Skogestad 1991a). At the end of the paper we consider the $D_{w} V$-configuration, and show that in this case there may exist solutions corresponding to stable limit cycles.

We only consider the multiplicity and instability caused by singularity in the input transformation. The multiplicity and instability that may be caused by including the energy-balance will have similar implications for the dynamics and control of distillation columns.

\section{Results on sieady-state multiplicity in ideal distillation}

We give a brief review of the results on multiplicity caused by singularities in the input transformation presented in Jacobsen and Skogestad 1991 a.

Consider the two-product distillation column in Fig. 1. If the feed to the column is given there are at least four flows that may be specified: reflux $L$, boilup $V$, distillate $D$

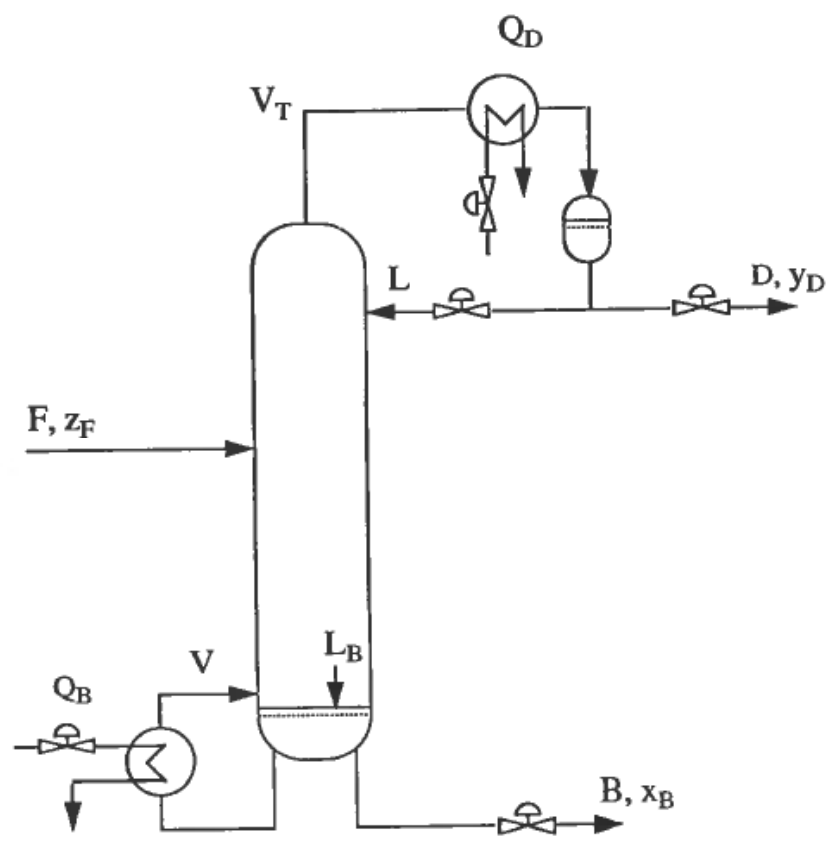

Figure 1. Two product distillation column. 
and bottoms flow $B$. However, for a given column there are only two degrees of freedom at steady-state, that is, only two of these flows may be specified independently. A specific choice of two independent variables is denoted a 'configuration'.

Jacobsen and Skogestad (1991a) provide an example of steady-state multiplicity in a column separating a mixture of methanol and $n$-propanol. The column has mass reflux and molar boilup as independent variables, i.e., $L_{w} V$-configuration. Data for the column are given in Table 1. Note that the energy balance is excluded, i.e., constant molar flows are assumed. Some steady-state solutions are given in Table 2, and we see that for a specification of mass reflux $L_{w}=50.0 \mathrm{~kg} / \mathrm{min}$ and molar boilup $V=2.0 \mathrm{kmol} / \mathrm{min}$ we have three possible solutions $I I, I I I$ and $I V$ in terms of compositions. The multiplicity is graphically illustrated in Fig. 2.

The observed mutliplicity is caused by the transformation between the actual flowrates (mass) and the molar flow-rates which determine separation. For a binary mixture the transformation between mass reflux, $L_{w}$, and molar reflux, $L$, is given by

$$
L=L_{w} / M ; \quad M=y_{D} M_{1}+\left(1-y_{D}\right) M_{2}
$$
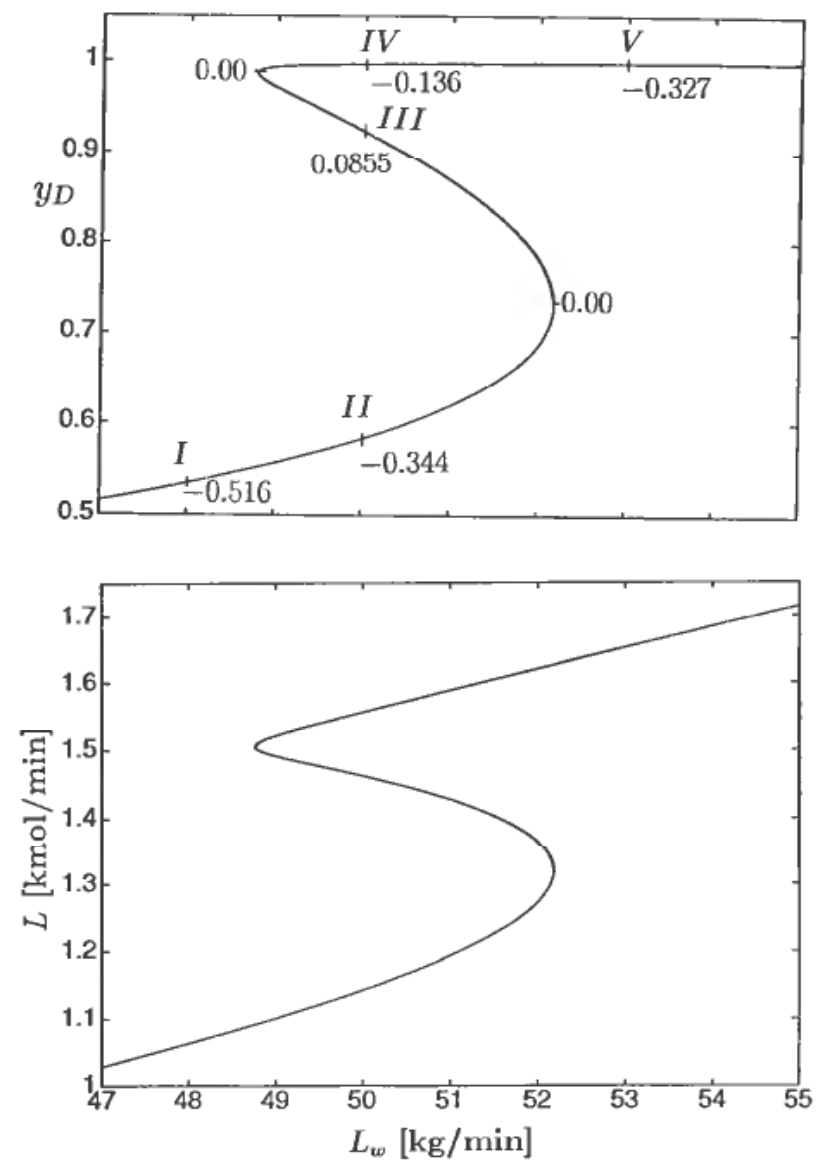

Figure 2. Methanol-propanol column with constant molar flows: Multiple steady-states for $L_{w} V$-configuration. Mass reflux $L_{w}$ is varied while molar boilup $V$ is fixed at $2.0 \mathrm{kmol} /$ $\min$. On the upper plot the corresponding maximum eigenvalue is shown at some of the steady-state solutions. 
Here $M_{i}$ denotes the molecular weight of the individual components. One might expect the molar reflux to increase monotonically with the mass reflux, that is, $\left(\partial L / \partial L_{w}\right)_{V}>0$. However, because $M$ is a function of composition, $y_{D}$, and thereby of $L_{w}$, this might not be the case. Assuming molar boilup $V$ fixed and differentiating $L_{w}=L M$ on both sides with respect to $L$ yields

$$
\left(\frac{\partial L_{w}}{\partial L}\right)_{V}=M+L\left(M_{1}-M_{2}\right)\left(\frac{\partial y_{D}}{\partial L}\right)_{V}
$$

For $M_{1}<M_{2}$, which is usually the case (the most volatile component has the smallest molecular weight), the second term on the right hand side of eqn. (2) will be negative and the total differential may take either sign. The transformation from $L_{w}$ to $L$ will be singular when $\left(\partial L_{w} / \partial L_{V}=0\right.$. A singular point will correspond to a limit point. Jacobsen and Skogestad (1991 a) state that solutions with $\left(\partial L_{w} / \partial L\right)_{V}<0$ (middle branch in Fig. 2) correspond to unstable operating points, but they do not prove this rigorously.

\section{Open-loop dynamics and instability for $L_{w} V$-configuration}

The maximum eigenvalue in selected operating points of the methanol-propanol column with the $L_{w} V$-configuration are shown in Fig. 2. From the figure we observe that the eigenvalues at the upper and lower branches are negative, implying stability, while those at the intermediate branch (negative slope) are positive, implying instability of the operating points. Note that the unstable operating points only have a single eigenvalue in the right half plane. The maximum eigenvalues at the singular points are zero as expected.

\subsection{Conditions for instability}

One-stage column. Consider the simple column in Fig. 3 with one theoretical stage (the reboiler) and a total condenser. Of course, such a column will never be operated in practice because the reflux is simply wasting energy and has no effect on separation. However, we analyse this column due to the simplicity of the dynamic model. As shown in Jacobsen and Skogestad (1991 a), even such a simple column with ideal thermodynamics may have multiple steady-state solutions.

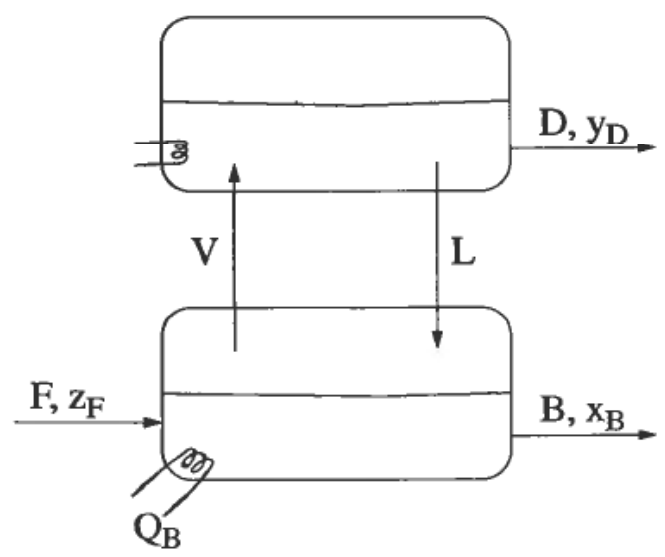

Figure 3. One-stage distillation column with total condenser. 
Assume binary separation, liquid feed, constant holdup in the reboiler $\left(M_{L}\right)$ and negiligible holdup in the condenser. The dynamic model of the column becomes:

$$
M_{L} \frac{d x_{B}}{d t}=F z_{F}-D y_{D}-B x_{B}
$$

We have $D=V-L$ and $D+B=F$ and with $L$ and $V$ as independent variables we get

$$
M_{L} \frac{d x_{B}}{d t}=F\left(z_{F}-x_{B}\right)+L\left(y_{D}-x_{B}\right)+V\left(x_{B}-y_{D}\right)
$$

Linearization, Laplace transformation and introduction of deviation variables assuming $F, z_{F}$ and $V$ constant yields

$$
s M_{L} \Delta x_{B}(s)=-D \Delta y_{D}(s)-B \Delta x_{B}(s)+\left(y_{D}-x_{B}\right) \Delta L(s)
$$

Assuming constant relative volatility $\alpha$ yields the following relation between $\Delta y_{D}(s)$ and $\Delta x_{B}(s)$

$$
\frac{\Delta y_{D}(s)}{\Delta x_{B}(s)}=\frac{\alpha}{\left(1+(\alpha-1) x_{B}\right)^{2}}=K\left(x_{B}\right)
$$

Equation (5) then becomes

$$
\Delta x_{B}(s)=\frac{y_{D}-x_{B}}{M_{L} s+a} \Delta L(s) ; a=K D+B
$$

As $a$ is always positive, the pole $-a / M_{L}$ is always negative, implying that all operating points are stable when molar reflux $L$ and molar boilup $V$ are independent variables.

Now consider mass reflux $L_{w}$ as an input instead of molar reflux $L=L_{w} / M$. By linearization we obtain for binary separations

$$
\Delta L=\frac{1}{M} \Delta L_{w}+L \frac{M_{2}-M_{1}}{M} K \Delta x_{B}
$$

Substituting eqn. (8) into eqn. (7) we obtain the following transfer-function between liquid composition, $\Delta x_{B}(s)$, and mass reflux $\Delta L_{w}(s)$ :

where

$$
\Delta x_{B}(s)=\frac{y_{D}-x_{B}}{M_{L} s+a_{w}} \frac{\Delta L_{w}(s)}{M}
$$

$$
a_{w}=K D+B-\left(y_{D}-x_{B}\right) \frac{M_{2}-M_{1}}{M} K L
$$

The operating point is unstable for $a_{w}<0$. At steady-state eqns (6) and (7) yield

$$
\left(\frac{\partial y_{D}}{\partial L}\right)_{V}=\frac{y_{D}-x_{B}}{D+B / K}
$$

and from eqn. (2) we find that instability $\left(a_{w}<0\right)$ is equivalent to a negative slope for $\left(\partial L_{w} / \partial L\right)_{v}$. This result is in accordance with numerical results.

Multistage column. Jacobsen and Skogestad (1991b) provide evidence for the instability of multistage columns. Using certain assumptions they show that the maximum eigenvalue $\lambda_{\max }^{L_{w} V}$ of a column operating with the $L_{w} V$-configuration is given by

$$
\lambda_{\max }^{L_{L} W V}=\lambda_{\max }^{L V}\left(1-\frac{\left(\partial y_{D} / \partial L\right)_{V} L\left(M_{2}-M_{1}\right)}{M}\right)
$$


Here $M$ is as defined previously and $\lambda_{\max }^{L V}$ is the maximum eigenvalue for the $L V$ configuration. Jacobsen and Skogestad (1991b) show that instability, $\lambda_{\max }^{L_{L} V}>0$, corresponds to having a negative slope between mass and molar reflux. For details we refer to Jacobsen and Skogestad (1991b).

\subsection{Effect of operating conditions on stability}

Jacobsen and Skogestad (1991a) provide analytical results on when a negative slope between mass and molar reflux, i.e., instability, is most likely. They show that a negative slope is most likely with large internal flows (i.e., large $L$ and $V$ ) and with intermediate purities in the top (i.e., intermediate $L$ for given $V$ ). This corresponds to having $L$ and $\left(\partial y_{D} / \partial L\right)_{V}$ large, and according to eqn. (12) this is the case for which instability is most likely. On this basis Jacobsen and Skogestad (1991a) show that there may exist three possible regimes of operation for distillation columns depending on the size of the internal flows:

- Internal flows low: Unique stable operating points.

- Internal flows intermediate: Multiple steady states, one of which is unstable.

- Internal flows high: Unique unstable operating points.

Note that the analytical treatment in (Jacobsen and Skogestad 1991a) was based on ideal separation with constant relative volatility and constant molar flows.

\section{Effect on multiplicity and instability on column operation}

It is obvious that the multiplicity and instability presented here may have implications for the operation of distillation columns using the $L_{w} V$-configuration. In manual (open-loop) operation the multiplicity may be experienced as small changes in the manipulated flows may cause sudden jumps in the product compositions. Furthermore, one will experience inability to reach a desired product composition if the separation corresponds to an unstable operating point. However, we show that an unstable operating point usually may be easily stabilized by feedback control.

\subsection{Manual operation}

We will again consider the methanol-propanol column in Table 1. Assume that the column initially operates with reflux $L_{w}$ fixed at $50.0 \mathrm{~kg} / \mathrm{min}$ and boilup $V$ fixed at $2.0 \mathrm{kmol} / \mathrm{min}$. For these values of $L_{w}$ and $V$ there are three possible solutions in terms of the product compositions according to Table 2 and Fig. 2. Only two of these are stable, namely operating points $I I$ and $I V$. Assume that the column just has been started up and that the product compositions corresponds to solution $I I$, i.e., $y_{D}=0.584$ and $x_{B}$ $=0.0035$, while the desired operating point is operating point $I I I$ in Table 2, i.e., $y_{D}$ $=0$-924. In order to increase the purity in the top the operator starts to increase reflux $L_{w}$ in a stepwise fashion. The reflux is increased with $0.5 \mathrm{~kg} / \mathrm{min}$ every $40 \mathrm{~min}$. This is illustrated in Fig. 4 together with the response in the top composition. In the beginning

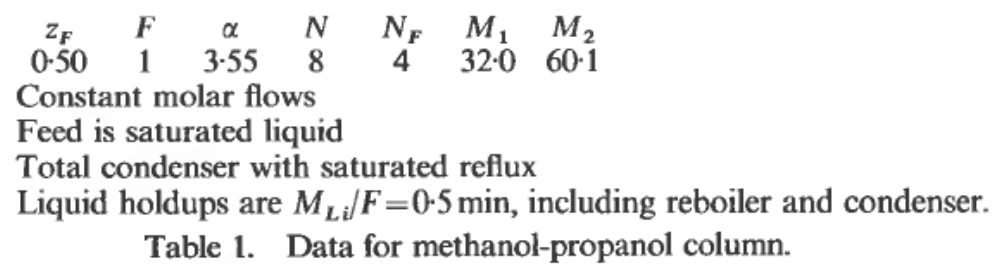




$\begin{array}{cccccc} & \begin{array}{c}L \\ \mathrm{kmol} / \mathrm{min}\end{array} & \begin{array}{c}D \\ \mathrm{kmol} / \mathrm{min}\end{array} & \begin{array}{c}L_{w} \\ \mathrm{~kg} / \mathrm{min}\end{array} & y_{D} & x_{B} \\ I & 1.064 & 0.936 & 48.00 & 0.534 & 3 \cdot 10 \mathrm{e}-3 \\ I I & 1.143 & 0.857 & 50.00 & 0-584 & 3 \cdot 50 \mathrm{e}-3 \\ I I I & 1.463 & 0.537 & 50.00 & 0.9237 & 7 \cdot 80 \mathrm{e}-3 \\ I V & 1.555 & 0.445 & 50-00 & 0.9969 & 0-104 \\ V & 1.650 & 0-350 & 53.00 & 0.9984 & 0-233\end{array}$

Table 2. Steady-state solutions for methanol-propanol column with $V=2 \cdot 0 \mathrm{kmol} / \mathrm{min}$ and $L_{w}$ in the range 48 to $53 \mathrm{~kg} / \mathrm{min}$.
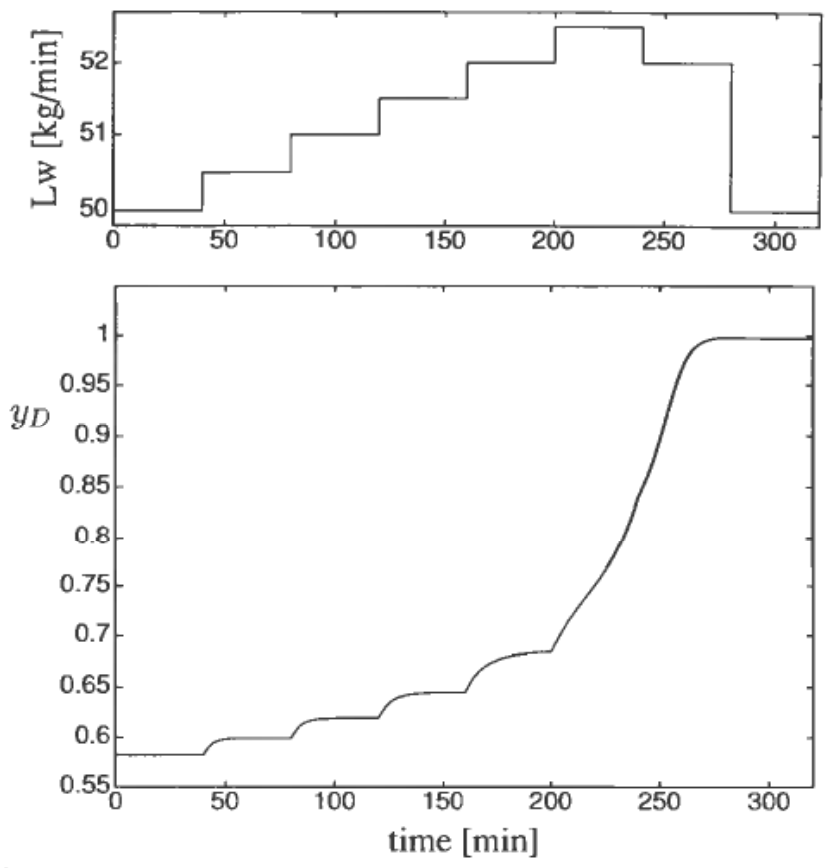

Figure 4. Nonlinear open-loop response of methanol-propanol column to changes in mass reflux $L_{w}$. Boilup $V=2.0 \mathrm{kmol} / \mathrm{min}$.

the top composition $y_{D}$ increases slightly with reflux as expected. However, as the operator increases the reflux from 52 to $52.5 \mathrm{~kg} / \mathrm{min}$ the top composition starts to increase drastically. The reason is that the reflux has been increased passed the lower singular point in Fig. 2, and the column goes through an instability known as a 'catastrophic jump'. The operator observes that the top composition becomes too pure and the bottom composition too impure, and reduces the reflux back to $52.0 \mathrm{~kg} / \mathrm{min}$. However, as seen from Fig. 4 this does not have the desired effect, and the operator decides to reduce the reflux all the way back to $50.0 \mathrm{~kg} / \mathrm{min}$. However, due to the multiplicity the column now settles in operating point $I V$ with $y_{D}=0.997$, that is, hysteresis is experienced in the operation.

The simulations in Fig. 4 demonstrate three different effects that may observed in columns with multiple steady states:

(1) inability to reach open-loop unstable operating points by manual operation,

(2) catastrophic jumps as the column goes through a singular point and

(3) hysteresis in operation. 


\subsection{Operation with composition control}

As we have seen, columns operating with mass or volume inputs may be open-loop unstable, and will require feedback control (in addition to level and pressure control) for stabilization. From control theory it is well known that unstable poles by themselves do not present any bandwidth limitations; on the contrary they put a lower limit on allowable bandwidth of the closed-loop system. Problems will therefore only arise if there are bandwidth limitations like right half plane zeros at frequencies comparable to the right half plane pole ('The system goes unstable before we are able to observe what is happening') or if there are constraints ('we cannot counteract the instability'). For plants with a RHP pole $p$ and a RHP zero $z$ one must in general require $p<z$ in order to be able to stabilize the column. With a limited structure of the controller, for example, a PI controller, the distance between $p$ and $z$ must be larger.

Good control of distillation columns usually requires two-point control, i.e., feedback control of both product compositions. However, in order to stabilize an openloop unstable column one-point control will suffice. This is also the way most industrial columns with composition control are operated. An unstable column operating with the $L_{w} V$-configuration may be stabilized by controlling either top or bottom composition, or any other variable related to composition, for example a temperature inside the column.

For operating point $I I I$ of the methanol-propanol column the RHP-pole is at $p=0.086 \mathrm{~min}^{-1}$ and we are unable to stabilize the column with a PI-controller when the deadtime exceeds $11 \mathrm{~min} .\left(z \approx 2 / \theta_{d}=0 \cdot 182 \mathrm{~min}^{-1}\right)$. However, composition measurements in industrial columns (GC-analysis) may typically have deadtimes up to $30 \mathrm{~min}$, and one should then use faster temperature measurements in order to stabilize the column.

Nonlinear Simulations. Figure 5 shows nonlinear simulations of the methanolpropanol column using a single-loop PI-controller ${ }^{1}$ between top composition $y_{D}$ and mass-reflux $L_{w}$ with a 1 minute measurement delay included. Molar boilup $V$ is kept constant at $2.0 \mathrm{kmol} / \mathrm{min}$. The figure shows the responses to setpoint changes in $y_{D}$ from operating point $I I$ (open-loop stable) to operating point $I I I$ (open-loop unstable) and then further on to operating point $I V$ (open-loop stable). A logarithmic measurement $Y_{D}=\ln \left(1-y_{D}\right)$ was used in the controller as this reduces the nonlinearity of the initial response between different operating points (Slogestad and Morari 1988). From the figure we see that the controller is able to stabilize the open-loop unstable operating point $I I I$ with a RHP pole at $0.086 \mathrm{~min}^{-1}$. The simulations also show that the same controller may be used in these three widely differing operating points. The reason is that the initial response (high-frequency dynamics) in terms of logarithmic composition $Y_{D}$ is similar in all operating points. We would get instability if we used mole fractions, $y_{D}$, as is done conventionally. From the plot of mass-reflux against time we see that the steady-state change in the input is zero, showing that the three operating points are multiple solutions.

One should be careful about detuning a controller in an open-loop unstable process as the bandwidth may become lower than the minimum allowable and the operating point becomes closed-loop unstable. This is illustrated in Fig. 6, where the controller gain has been reduced by a factor of two compared to the simulations in Fig. 5 . Operating point $I I I$ is now closed-loop unstable, and a small setpoint change makes

${ }^{1}$ Tuned to yield reasonably fast response. Note that Ziegler-Nichols tuning rules resulted in a closed-loop unstable system. 

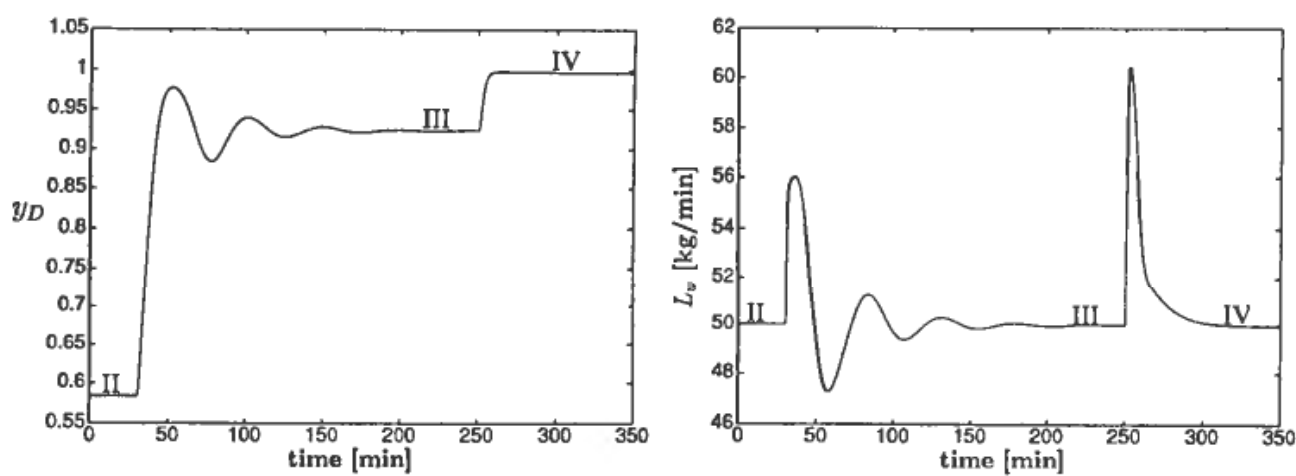

Figure 5. Nonlinear simulation of methanol-propanol column with one-point control of topcomposition $y_{D}$ using mass reflux $L_{w}$. Setpoint changes from operating point $I I$ to $I I I$ and from $I I I$ to $I V$. Boilup $V=2.0 \mathrm{kmol} / \mathrm{min}$. Controller parameters: $k=3.0$ and $\tau_{I}=11.0 \mathrm{~min}$. Gain is for logarithmic composition, $\log \left(1-y_{D}\right)$.
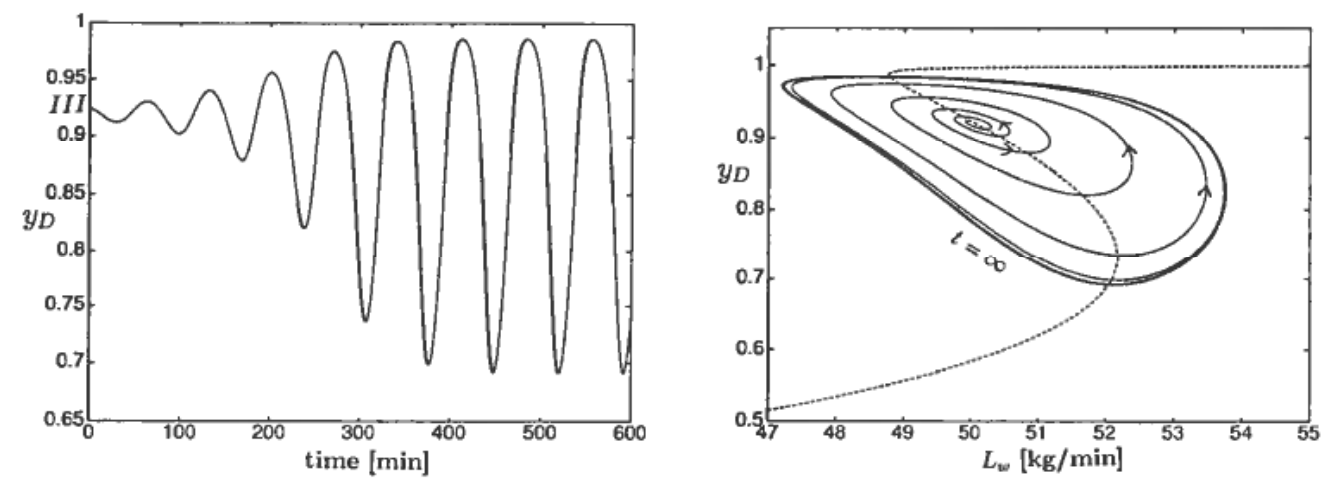

Figure 6. Nonlinear simulation of methanol-propanol column with one-point control of topcomposition $y_{D}$ using mass reflux $L_{w}$ Controller gain reduced by a factor of 2 compared to Fig. 5. Left plot: Time as independent variable. Right plot: Phase-plane plot. Dashed line shows steady-state solutions.

the system start drifting away. However, this does not imply that the column goes globally unstable in the sense that physical constraints are violated. Since there exists steady-state solutions above and below the unstable solution the column goes into a stable limit cycle. If the controller gain is reduced further the limit cycle will continue, but now with a longer period of each cycle and with higher peaks in composition. There will also exist cases where there are no solutions either above or below the unstable solution. In this case the column is likely to go globally unstable as either the condenser (missing upper branch) or reboiler (missing lower branch) would run dry.

For a discussion of the implications of open-loop instability for two-point control we refer to Jacobsen and Skogestad (1991b).

\section{Other bifurcation parameters}

So far we have only considered the manipulated inputs, for example, reflux $L_{w}$ and boilup $V$, as potential bifurcation parameters. That is, in all studies we have assumed the other parameters, feed flow $F$, feed composition $z_{F}$, feed liquid fraction $q_{F}$, tray 
efficiency and so on to be fixed. However, it is clear that these parameters will vary during operation and may, similarly to the inputs, cause the column operation to go from open-loop stable to open-loop unstable.

To illustrate this consider Fig. 7 which shows steady-state solutions for the methanol-propanol column with $L_{w}=50.0 \mathrm{~kg} / \mathrm{min}, V=2.0 \mathrm{kmol} / \mathrm{min}$ and feed composition $z_{F}$ in the range 0.40 to 0.60 . From the figure we see that there are multiple solutions for $z_{F}$ in the range 0.46 to 0.54 . This implies that disturbances in the feed composition may cause the column to go through a singular point and thereby 'jump' to another solution branch. This is illustrated in Fig. 8 which shows the response in top composition $y_{D}$ to a change in feed composition $z_{F}$ from 0-50 (operating point $I V$ in Table 2) to 0.46. The figure illustrates how the top composition 'jumps' to the lower solution branch and settles in operating point $V I$. When the feed composition returns to $z_{F}=0.50$ the solution remains on the lower branch and settles in operating point $I I$.

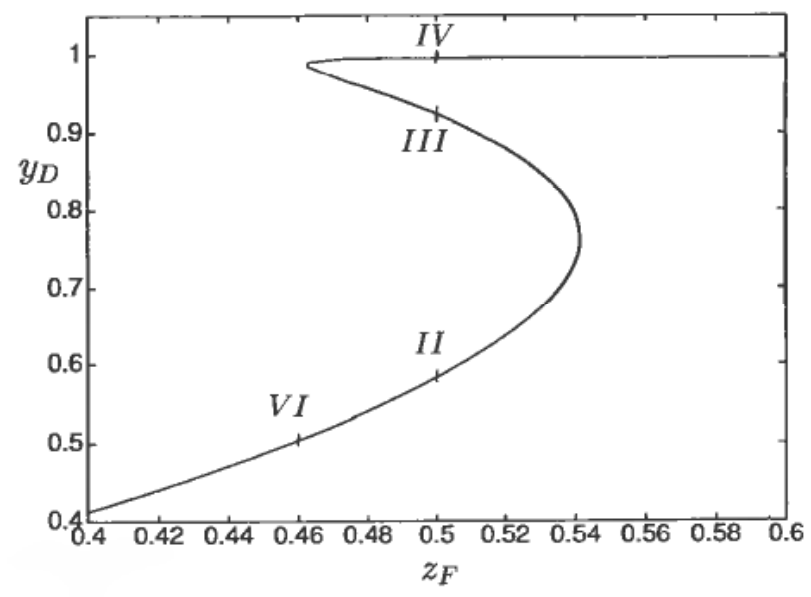

Figure 7. Steady-state solutions as a function of feed composition $z_{F}$ for methanol-propanol column. Reflux $L_{w}=50 \mathrm{~kg} / \mathrm{min}$, Boilup $V=2 \cdot 0 \mathrm{kmol} / \mathrm{min}$.

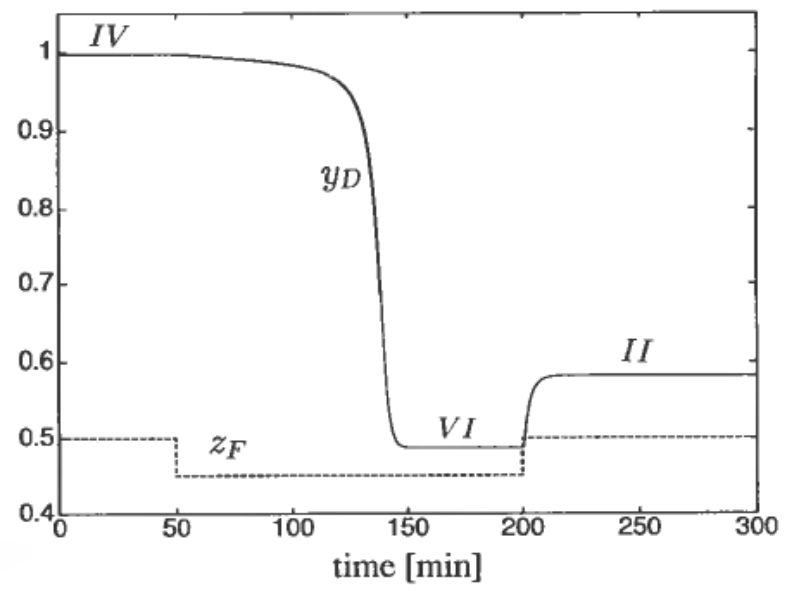

Figure 8. Nonlinear open-loop simulation of methanol-propanol column for changes in $z_{F}$. Reflux $L_{w}=50 \mathrm{~kg} / \mathrm{min}$ and boilup $V=2.0 \mathrm{kmol} / \mathrm{min}$. Roman numbers $I I-I V$ refer to Table 2 with $z_{F}=0 \cdot 50$. Operating point $V I$ corresponds to $z_{F}=0 \cdot 46$. 


\section{Instability with the $D_{w} V$-configuration}

We have so far only considered using reflux and boilup as independent variables, i.e. the $L_{w} V$-configuration. This is also the most widespread configuration in industry. However, there are many different configurations that may be used. For instance, changing condenser level control from using distillate $D_{w}$ to using reflux $L_{w}$ results in the $D_{w} V$-configuration. For all the examples we have studied this configuration yields a unique steady-state solution in terms of compositions. Furthermore, we have assumed perfect level control, in which the operating point is found to be asymptotically stable. However, here we show that without the assumption of perfect level control the operating point may become unstable also with the $D_{w} V$-configuration. We start by considering an example and will then explain the results thereof using analytical results.

Example. We will again consider the methanol-propanol column in Table 1. The holdups in the reboiler and condenser are increased to $M_{D} / F=M_{B} / F=5.0 \mathrm{~min}$. We consider the case with constant molar flows, and use distillate flow $D_{w}$ and boilup $V$ as independent inputs, i.e., $D_{w} V$-configuration. With this configuration the condenser level is controlled by reflux $L_{w}$ and the reboiler level is controlled by bottoms flow $B_{w}$. The nominal operating point we consider has $D_{w}=18.36 \mathrm{~kg} / \mathrm{min}$ and $V=2.0 \mathrm{kmol} / \mathrm{min}$. For these specifications we obtain $y_{D}=0.9237$ and $x_{B}=0.0078$, and the steady-state is unique. Note that the operating point corresponds to solution $I I I$ for the $L_{w} V$ configuration in Table 2 and Fig. 2, that is, the operating point is unstable with reflux and boilup as independent variables.

We now consider the stability of the operating point for different gains $K_{M_{D}}$ in the condenser level controller. A pure proportional controller is used, $d L_{w}(s)$ $=K_{M_{D}} d M_{D_{w}}(s)$. We assume perfect level control in the reboiler. Figure $9(a)$ shows the response in top composition to a small increase in $D_{w}$, keeping $V$ constant, with level control gain $K_{M_{D}}=0.05$. We see that the response is oscillatory but asymptotically stable, i.e., a stable spiral. Figure $9(b)$ shows the corresponding response with $K_{M_{D}}$ reduced to 0.03 , and we see that the steady state now is an unstable spiral. However, the response settles into a stable periodic solution, that is, a stable limit cycle.

The fact that the steady-state changes from a stable spiral to an unstable spiral as the level control gain is reduced implies that a pair of complex conjugate eigenvalues cross the imaginary axis. The fact that a stable limit cycle appears as the steady-state becomes unstable, implies that the system goes through a dynamic bifurcation known as the Hopf bifurcation.

\subsection{Analytical treatment}

To understand why the steady-state for the $D_{w} V$-configuration becomes unstable, consider the transfer function $\left(\partial y_{D} / \partial D_{w}\right)_{V}(s)$ which may be written

$$
\left(\frac{\partial y_{D}}{\partial D_{w}}\right)_{V}(s)=\left(\frac{\partial y_{D}}{\partial L_{w}}\right)_{V}(s)\left(\frac{\partial L_{w}}{\partial D_{w}}\right)_{V}(s)
$$

Here the transfer function $\left(\partial y_{D} / \partial L_{w}\right)_{V}(s)$ expresses the effect of reflux on top composition with the $L_{w} V$-configuration, and we have seen that it may be unstable with a single RHP pole. For simplicity we consider only the largest pole in the transfer function

$$
\left(\frac{\partial y_{D}}{\partial L_{w}}\right)_{V}(s)=\frac{k}{s-a}
$$




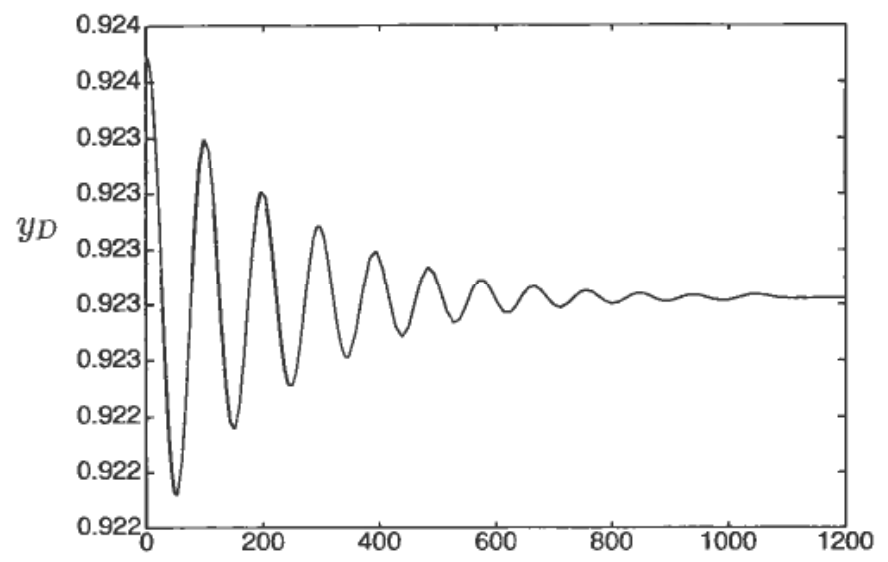

(a)

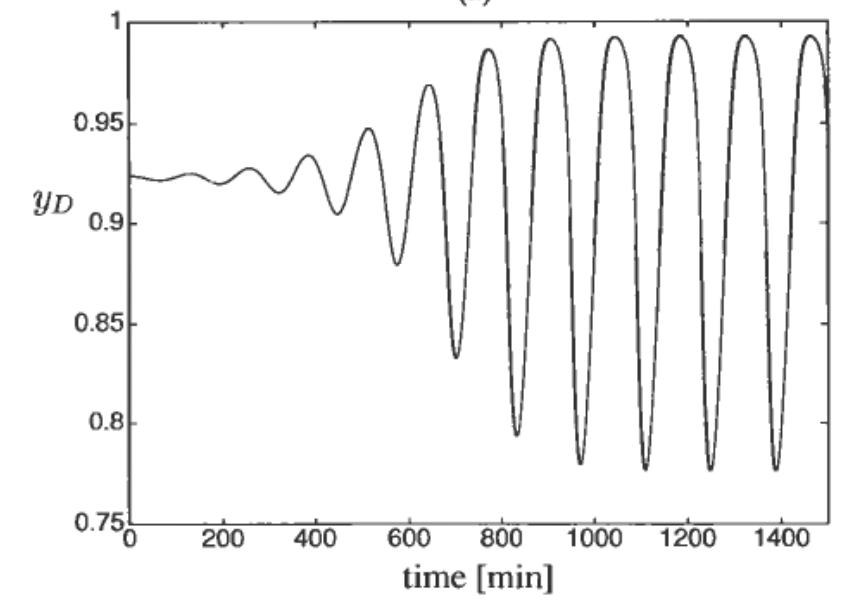

(b)

Figure 9. Nonlinear open-loop simulation of methanol-propanol column with $D_{w} V$ configuration. Responses to small increase in $D_{w}$ with different gains $K_{M_{D}}$ in condenser level controller. (a) $K_{M_{D}}=0.05$. (b) $K_{M_{D}}=0.03$. Boilup $V=2.0 \mathrm{kmol} / \mathrm{min}$.

Here $a$ denotes the maximum eigenvalue for the $L_{w} V$-configuration. The transfer function $\left(\partial L_{w} / \partial D_{w}\right)_{V}(s)$ may be computed from a material balance around the condenser

$$
d L_{w}(s)=\frac{K_{M_{v}}}{s}\left(d V_{T_{w}}(s)-d L_{w}(s)-d D_{w}(s)\right)
$$

Differentiation of (15) yields

$$
\left(\frac{\partial L_{w}}{\partial D_{w}}\right)_{V}(s)=\frac{K_{M_{D}}}{K_{M_{D}}+s}\left(V_{T}\left(M_{1}-M_{2}\right)\left(\frac{\partial y_{T}}{\partial D_{w}}\right)_{V}(s)-1\right)
$$

Here $y_{T}$ denotes the composition of $V_{T}$. We assume negligible condenser holdup so that $\left(\partial y_{T} / \partial D_{w}\right)_{V}(s)=\left(\partial y_{D} / \partial D_{w}\right)_{V}(s)$. Inserting eqns (14) and (16) into eqn. (13) yields

$$
\left(\frac{\partial y_{D}}{\partial D_{w}}\right)_{V}(s)=\frac{K_{M_{D}} k}{s^{2}+\left(K_{M_{D}}-a\right) s-K_{M_{D}}\left(a+k V_{T}\left(M_{1}-M_{2}\right)\right)}
$$


The poles of the transfer function (17) become

$$
\lambda_{1,2}=-\frac{1}{2}\left(K_{M_{D}}-a\right) \pm \frac{1}{2}\left(\left(K_{M_{D}}-a\right)^{2}+4 K_{M_{D}}\left(a+k V_{T}\left(M_{1}-M_{2}\right)\right)\right)^{1 / 2}
$$

Let us now use eqn. (18) to consider the stability of the $D_{w} V$-configuration for the two cases when the pole $a$ of the $L_{w} V$-configuration is in the LHP and RHP, respectively:

(1) Stable $L_{w} V$-configuration, $a<0$ : In this case the first term in eqn. (18) is negative for all values of $K_{M_{D}}>0$. Furthermore, the second term under the root in eqn. (18) is negative and the root will be real with a value less than $\left(K_{M_{D}}-a\right)$ or it will be imaginary. This implies that both eigenvalues in eqn. (18) are in the LHP, that is, the $D_{w} V$ configuration is stable for all values of $K_{M_{D}}>0$.

(2) Unstable $L_{w} V$-configuration, $a>0$ : In this case we have that the first term in eqn. (18) is positive if $K_{M_{D}}<a$, that is, at least one of the eigenvalues in eqn. (18) are in the RHP with $K_{M_{D}}<a$. The size of $K_{M_{D}}$ will determine whether the root in eqn. (18) is imaginary. For $K_{M_{D}}=a$, i.e., the bifurcation point, we have that the root is imaginary if $k V_{T}\left(M_{1}-M_{2}\right)<-a$, which is the case in all examples we have studied.

We conclude from the above analysis that a prerequisite for instability with the $D_{w} V$-configuration is that the operating point is unstable with the $L_{w} V$-configuration. This is not too surprising as the level control for the $D_{w} V$-configuration may be viewed as a feedback effect on the $L_{w} V$-configuration. If the feedback control is not tight enough, we are not able to stabilize the column, which is similar to what we found for the case of one-point control with the $L_{w} V$-configuration. With a gain $K_{M_{D}}=0$, i.e., no condenser level control, we see from eqn. (18) that there will be a RHP pole at $a$ (in addition to a pole at 0 ), and we effectively have the stability properties of the $L_{w} V$ configuration.

In our example we find that the $L_{w} V$-configuration is unstable with a pole $a=0.047$ (with $M_{D} / F=M_{B} / F=5 M_{B} / F=5 \mathrm{~min}$ ) and from eqn. (18) we predict instability for the $D_{w} V$-configuration with $K_{M_{D}}<0.047$. From the full model we find that instability occurs for $K_{M_{D}}<0.043$. The deviation in predicted and computed value is explained by our assumptions of first-order response in eqn. (14) and neglibible condenser holdup in the analytical treatment.

\section{Conclusions}

Distillation columns operating with the $L_{w} V$-configuration may have multiple steady states and unstable operating points. The probability of instability increases with increased internal flows.

The multiplicity and instability may cause problems for manual operation of distillation columns. Three effects may be observed:

(1) catastrophic jump phenomena,

(2) inability to reach certain separations and

(3) hysteresis in operation.

An open-loop unstable operating point may usually be easily stabilized by feedback control of a column composition or temperature. If the bandwidth of the controller is not sufficiently tight the column may go into a stable limit cycle.

Columns operating with the $D_{w} V$-configuration (or the $L_{w} B_{w}$-configuration) may become unstable if the level control is not sufficiently tight. A stable limit cycle appears as the operating point becomes unstable. 


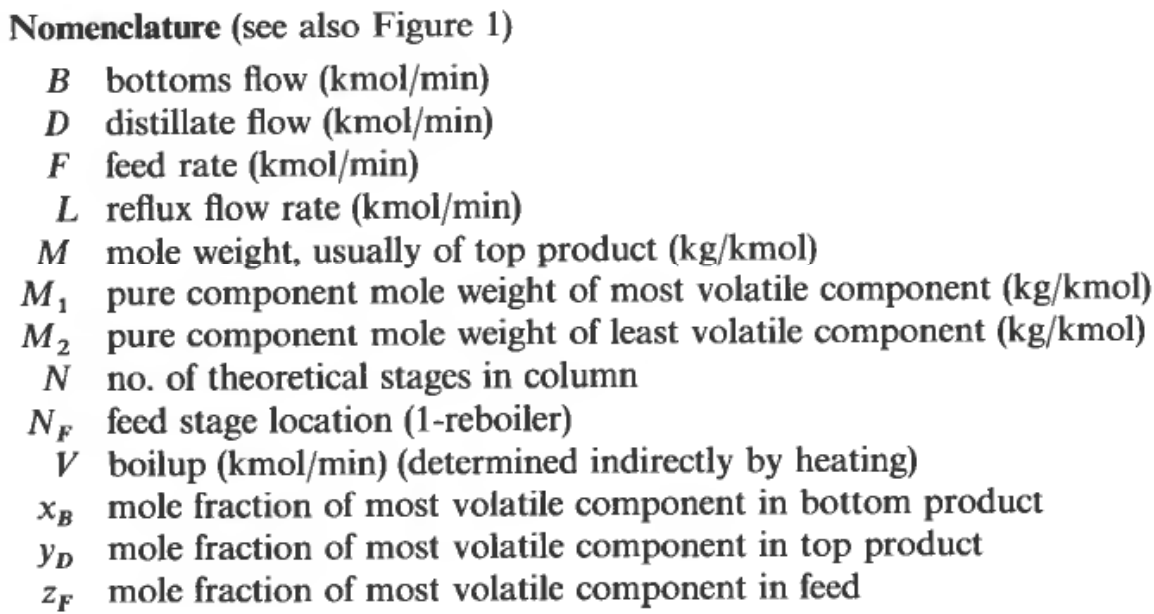

Greek symbols

$$
\alpha=\frac{y_{i} / x_{i}}{\left(1-y_{i}\right) /\left(1-x_{i}\right)} \text { relative volatility (binary mixture) }
$$

$\lambda_{\max }=\max _{i} \lambda_{i}(A)$ maximum eigenvalue

\section{Subscripts}

$w$ flow rate in $\mathrm{kg} / \mathrm{min}$

\section{REFERENCES}

Acrivos, A. and Amundson, N. R. (1955). Application of matrix mathematics to chemical engineering problems. Ind. Eng. Chem., 47, 1533-1541.

Doherty, M. F. and Perkins, J. D. (1982). On the dynamics of distillation processes-IV. Uniqueness and stability of the steady state in homogeneous continuous distillation. Chem. Eng. Sci., 37, 381-392.

JACobsen, E. W. and Skogestad, S. (1991a). Multiple steady states in ideal two-product distillation. AIChE J., 37, 499-511.

Jacobsen, E. W., and Skogestad, S. (1991b). Control of unstable distillation columns. Proc. of 1991 American Control Conference, Boston.

LAPIDUS, L. and AmUNDSON, N. R. (1950). Stagewise absorption and extraction equipmenttransient and unsteady state operation. Ind. Eng. Chem., 42, 1071-1078.

RosenBroCK, H. H. (1960). A theorem of 'Dynamic Conservation' for distillation, Trans. Instn. Chem. Engrs., 38, 279-287.

ROSENBROCK, H. H., (1962). A Lyapunov function with applications to some nonlinear physical problems. Automatica, 1, 31-53.

SKogestad, S. and MORARI, M. (1988). Understanding the dynamic behavior of distillation columns. Ind. \& Eng. Chem. Res., 27, 1848-1862.

SRIDHAR, L. N. and LUCIA, A. (1989). Analysis and algorithms for multistage separation processes. Ind. \& Eng. Chem. Res., 28, 793-803. 